\title{
Ethical considerations for genetic testing in the context of mandated cardiac screening before athletic participation
}

\author{
Emma F. Magavern, MD1, Lauren Badalato, MD1, Gherardo Finocchiaro, MD² and Pascal Borry, PhD'
}

The subject of cardiac screening before participation in competitive sports as a preventive strategy for sudden cardiac death has been debated at length by the international medical community. ${ }^{1}$ The role of genetics has been raised again in this context as a result of recent advances in molecular autopsy, with implications for family screening. There is currently widespread cardiac screening before athletic participation on a national level in the United States, Israel, and Italy, as well as within high-level sports organizations. ${ }^{2}$ It is mandated by sports organizations such as the Fédération Internationale de Football Association and the Premier League. Consider the case of an asymptomatic professional soccer player in his or her 20s. What role should genetic testing have, ethically, in the management of such an athlete in the presence of a family history of sudden cardiac death or an abnormal screening result? It is important to note that national screening programs involve the further complexity of screening minors, which raises an additional set of ethical considerations outside of the scope of this analysis.

Genetic testing has increasingly been incorporated into the mainstream diagnostic process and may be utilized in the presence of family history suggestive of inherited cardiac disease or borderline pathology noted on screening. ${ }^{3}$ The much discussed topic of cardiac screening before athletic participation has not included adequate discussion of underlying ethical concerns regarding the application of genetic testing in these circumstances, particularly those relevant to mandatory screening resulting in an eligibility decision. It is impossible to exclude this issue because many conditions sought, on the phenotypic level, by preparticipation screening are genetic.

\section{PROBLEMS WITH GENETIC TESTING IN THIS CONTEXT}

Phenotypes are highly variable and genotype does not always predict age of onset or severity. The penetrance and expressivity are variable, and a wide array of potential mutations cause the heterogeneous group of cardiovascular disorders that can predispose to sudden cardiac death (variants of unknown significance represent a further complication). As Richard et al. note, there are: "dramatic differences in...severity of ECG/echocardiography features, risk of complications and response to medical treatment." ${ }^{3}$ Although there is evidence of exercise causing harm in an athlete with a genetic predisposition to arrhythmogenic right ventricular cardiomyopathy, there is no evidence basis for disqualification recommendations for athletes who are genotype-positive and phenotypenegative for hypertrophic cardiomyopathy, Marfan syndrome, Brugada, catecholaminergic polymorphic ventricular tachycardia, long and short QT, dilated cardiomyopathy, or left ventricular noncompaction owing, at least in part, to our very limited knowledge of genetic variant pathogenicity. Thus, expert opinion-based disqualification recommendations for medical practitioners differ greatly regarding eligibility/disqualification of individuals who are genotype-positive and phenotype-negative. ${ }^{4}$

\section{MEDICAL DISQUALIFICATION RECOMMENDATIONS}

The American guidelines have been, in general, less restrictive than the European Society of Cardiology guidelines. ${ }^{4}$ Although the American Bethesda guidelines were recently updated in 2015, their recommended approach to genotype-positive, phenotype-negative individuals has not changed. The Bethesda guidelines require a threshold of phenotypic expression before disqualification is suggested, but the European Society of Cardiology guidelines recommend disqualification based on the finding of a pathogenic mutation alone for conditions such as Marfan syndrome or hypertrophic cardiomyopathy. ${ }^{4}$ However, the highly variable penetrance and expressivity of many such mutations complicate their use in risk evaluation. ${ }^{3}$ Furthermore, it is unclear where the line is between preparticipation clearance and discrimination in an employment context; for example, it is uncertain when (and where) a disqualification based on a hypertrophic cardiomyopathy diagnosis could be considered discrimination and how this would differ if based on genetic testing results versus a phenotypic manifestation only. The risk posed by competitive athletic activity may vary significantly based on the condition and specific genetic mutation, as well as the type of sport played. ${ }^{3}$

${ }^{1}$ Centre for Biomedical Ethics and Law, Department of Public Health and Primary Care, University of Leuven, Leuven, Belgium; ${ }^{2}$ Cardiovascular Research Centre, St. George’s University of London, London, UK. Correspondence: Emma F. Magavern (emma.magavern@gmail.com) 


\section{DISCRIMINATION?}

Is athletic "disqualification" a misleading designation in the context of a professional athlete? Is it not a euphemism for loss of employment? Considering the laws enacted by many nations barring genetic testing during pre-employment assessment (the Genetic Information Nondiscrimination Act in the United States), the boundary between acceptable preparticipation screening and employment discrimination is unclear in the context of professional athletics. A much discussed example of occupation modifiable risk is the case of alpha-1 antitrypsin, "the only disease for which the Equal Employment Opportunity Commission ruled that genetic discrimination has occurred." ${ }^{\prime}$ People with this genetic condition are strongly predisposed to emphysema, and this can be worsened exponentially by smoking or work that involves exposure to microscopic particles, such as construction. ${ }^{6,7}$ As a society, we cannot force such individuals to refrain from smoking; however, if this damage is caused by modifiable employment circumstances, then there is a strong argument to be made for testing.

Although some "soft paternalism" is accepted in the workplace (e.g., occupational health personnel can require safety goggles or gloves for employees' protection), one should question whether these protective measures should extend to a screening process that may culminate with genetic testing and, naturally, the conundrum of genotype-positive, phenotypenegative conditions. This is a setting in which genetic testing could result in high false-positive and false-negative rates, as noted by Driest et al. in the context of arrhythmia-related genetic variants. ${ }^{8}$ Downstream testing can be resource-intensive and invasive, without a clear promise of benefits.

\section{CONCLUSIONS}

The tendency for patients to feel pressured into diagnostic testing downstream of routine screening has been previously described as a type of "screening trap." Although downstream genetic testing would not be mandated, informed consent may be coercive in the context of requisite preparticipation screening because further diagnostics may contribute to the "eligibility" assessment necessary for employment as a professional athlete.

In the majority of clinical cases, a genetic test would not be performed in the absence of a phenotypic manifestation. However, because the first manifestation of the conditions uncovered by preparticipation screening is a possibly fatal cardiac event, the threshold to recommend testing may be low in the presence of a family history, particularly in settings where physicians are legally liable for eligibility.

Therefore, there should be a diagnostic delineation prior to genetic testing downstream of any mandated cardiac screening in this context; certainly, separate informed consent should be sought. In light of the international sports arena, differing international legislation, and inconsistent medical disqualification recommendations to physicians, it may be prudent to suggest confidential professional genetic counseling and appropriate subsequent testing from a medical practitioner external to the screening process.

\section{THE ETHICOLEGAL POLICY GAP AND THE WAY FORWARD}

Appropriate counseling should acknowledge that the diagnosis of a genetic condition can have psychosocial implications. In a cohort of athletes diagnosed with serious cardiac conditions, Asif et al. documented increased metrics of psychological distress in a subpopulation diagnosed with a hereditary condition as compared with the rest of the group..$^{10}$ Additionally, significantly worse psychological impact scores were recorded in those permanently disqualified from sports compared with those prescribed some or no restriction to activity. ${ }^{10}$ Furthermore, there may be implications for practical concerns such as life and disability insurance. Given these potential harms, there is an ethical need for a dominant role of patient autonomy in the outcome of any mandated preparticipation cardiac screening, particularly with regard to genetic testing. The role of the genetic counselor is vital to this process.

It remains fundamental to address the athletic population's autonomy in having the "right not to know." As stated in the Dutch national screening report, "Respect for autonomy means that participants should wherever possible not be confronted with results that they would rather not have had."' One could reasonably argue that given the potential to prevent fatal cardiac events triggered by extreme exertion, the use of all possible diagnostic tools to identify at-risk individuals, including genetic testing, is justified. However, to best support patient autonomy, informed consent should be of high quality and there should be evidence demonstrating that such testing is clinically recommendable for that particular individual based on a thorough balancing of the possible risks and benefits. The ability to modify risk factors and to treat the condition tested for should be considered.

To date, there is no published literature that describes the ethical place of genetic testing in mandated preparticipation cardiac screening specific to scenarios leading to a medical eligibility decision; thus, it is vital to discuss these issues in public forums. Although genetic testing represents a valuable diagnostic tool, it is crucial that the role of genetic tests as follow-up to mandated screening be further clarified from an ethicolegal perspective. Furthermore, the use of these genetic tests must be in keeping with genetic data protection legislation as well as wider societal norms regarding limitations to medical information that an employer is able access or require.

Considering the currently incomplete regulatory framework, it may be prudent for any genetic testing recommended in the context of mandatory cardiac screening to be performed confidentially by an independent clinical genetics team (as a process separate from the screening and from the eligibility determination), with genetic counseling detailing the implications of testing. In this way, genetic testing could not be imposed and the results of genetic tests would not be used for eligibility decisions regarding professional athletes. 


\section{ACKNOWLEDGMENTS}

E.F.M. and L.B. were funded by Erasmus Mundus Master of Bioethics Fellowships. G.F. is funded by a research grant from the charitable organization Cardiac Risk in the Young (CRY).

\section{DISCLOSURE}

The authors declare no conflict of interest.

\section{REFERENCES}

1. Maron BJ. Historical perspectives on sudden deaths in young athletes with evolution over 35 years. Am J Cardiol 2015;116:1461-1468.

2. Maron BJ, Friedman R, Caplan A. Ethics of preparticipation cardiovascular screening for athletes. Nat Rev Cardiol 2015;12:1-4.

3. Richard P, Denjoy I, Fressart V, Wilson MG, Carré F, Charron P. Advising a cardiac disease gene positive yet phenotype negative or borderline abnormal athlete: is sporting disqualification really necessary? Br J Sports Med 2012;46 Suppl 1:i59-i68.
4. Pelliccia A, Zipes DP, Maron BJ. Bethesda Conference \#36 and the European Society of Cardiology Consensus Recommendations Revisited. A Comparison of U.S. and European Criteria for Eligibility and Disqualification of Competitive Athletes With Cardiovascular Abnormalities. J Am Coll Cardio/ 2008;52:1990-1996.

5. Klitzman R. The impact of social contexts in testing for alpha-1 antitrypsin deficiency: the roles of physicians and others. Genet Test Mol Biomarkers 2009;13:269-276.

6. Zuo L, Pannell BK, Zhou T, Chuang CC. Historical role of alpha-1-antitrypsin deficiency in respiratory and hepatic complications. Gene 2016;589:118-122.

7. Lurati AR. Occupational risk assessment and genetic testing in the workplace. Occup Med Heal Aff 2014;2:146.

8. Van Driest SL, Wells QS, Stallings S, et al. Association of arrhythmia-related genetic variants with phenotypes documented in electronic medical records. JAMA 2016;315:47.

9. Health Council of the Netherlands. Screening: Between Hope and Hype. The Hague; 2008.

10. Asif IM, Price DE, Ewing A, Rao AL, Harmon KG, Drezner JA. The impact of diagnosis: measuring the psychological response to being diagnosed with serious or potentially lethal cardiac disease in young competitive athletes. Br J Sports Med 2016;50:163-166. 